\title{
Optimal placement and sizing of distributed generation for loss minimization using ABC optimization
}

\author{
Madhu Valavala \\ Department of Electrical and Electronics Engineering, Sri Satya Sai University of Technology and Medical Sciences, \\ Sehore, India
}

\section{Article Info \\ Article history: \\ Received Jun 15, 2021 \\ Revised Jul 23, 2021 \\ Accepted Aug 20, 2021 \\ Keywords: \\ Artificial bee colony Distributed generation Optimization techniques Reactive power Voltage Regulation}

\begin{abstract}
The distributed generation (DG) refers to the use of the nearby units of small generation or in the sleefelzentres. Revisions have shown that unfitting choice of the position and scope of the DG can lead to bigger system sufferers than DG's sufferers. Public services are already available before the high loss of energy loss and of the mediocre voltage profile, in particular developing countries cannot tolerate any increase in losses. From optimal allocation, public services in the reduction of system losses improve tension adjustment and improve delivery reliability. This article aims to minimalize the annual system's annual loss through the appropriate positioning and size of the DG units. The artificials bee colony (ABC) of EPA are inspired by the behavior of the API feeding, this method is incredibly conventional, a stroke or a peoplated stochastic optimization algorithm to meet the solution of the specified problem. At MATLAB, a probabilistic approach is simulated to reach the target indicates that the size of the DGs to be installed to reduce almost the same loss as a percentage that the situation in which the load power is considered constant.
\end{abstract}

This is an open access article under the CC BY-SA license.

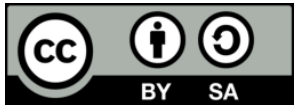

\section{Corresponding Author:}

Madhu Valavala

Department of Electrical and Electronics Engineering

Sri Satya Sai University of Technology and Medical Sciences

SH-18, Bhopal-Indore Road, Opp.Oil Fed Plant (M.P), Pachama, Madhya Pradesh, India

Email:vmadhu.eee@gmail.com

\section{INTRODUCTION}

Electrical distribution system is one in every of the integral parts of grid. It's answerable for delivering power to the consumers as economically and reliably as possible. The electrical grid is developing from a system with large centralized generators into a system with smaller generators interconnected at lower voltage levels. The facility flow is thus changed from centralized into bidirectional power flow. Energy resources interconnected within the MV or LV distribution grid is thought as distributed generation (DG) [1]. Other common names are decentral, dispersed or embedded generation. Some distributed generators apply sustainable energy, i.e., micro-turbines (biogas, optimized use of fuel, CHP), alternative energy and photovoltaic. These energy resources facilitate to adjust to the Kyoto protocol [2]-[5]. Distributed generation also increase the degree of produced power which can be the answer to fulfill increased power consumption within the society. By generating power locally, the energy efficiency increases thanks to reduced transmission losses. The installation process is shorter for DG than for big centralized plants, like atomic power plants. Customers are given the chance to provide their own electricity so as to cut back their energy expenses. The situation of the distributed energy resource (DER) has thus many impacts. By injecting power locally, the voltage gains in this interconnection point. This will atone for voltage sags but may additionally 
cause over-voltage problems. The assembly rate of distributed generation might in some cases have correlation to the climatic conditions [6]. The ability generated from wind depends of the wind speed and therefore the production rate from a solar cell depends on the sun irradiation. During weather conditions, the warmth production may increase and in CHP plants the facility production then also increases. A particular unpredictability of distributed generated power is introduced thanks to the weather correlation.

Every day, a deterministic analysis of the charge flow is performed to examine the appearance and operation of the grid. In order to calculate the system states and performance currents, the deterministic load current uses the defined values of power generation and features of the goods of a particular network configuration [7]. The accumulation of DG units to the distribution system, the system with Hitormiss and HitiTimity is injected [8]. In totaling of the uncertain energy of the injected DG, there are many more uncertainties in an extreme grid in an extreme grid, because of: (i) Volatility of demand for a fee, (ii) Changes in network configuration, (iii) Generator error, (iv) The DG units inject intermittent electricity in the grid.

As a result, the deterministic approach is not sufficient to investigate the structure of the fashion, and therefore, the results of a deterministic load stream can provide a non-realistic evaluation of the structure presentation [9]-[12]. For operational preparation issues, such as the valuation of the consistency of system arrangement or the strategy of the latest links, it is required to evaluate the road currents of spectrum of costs. For practical problems, it is not possible to contain individual load streams for each load change for the following reasons: (i) Prohibition credit of calculations. Load currents are required for the network with node and by different load values at each node, (ii) Difficulties to investigate and manufacture the outcomes of several load currents.

To request uncertainty, mathematical approaches are often used, such as the "probabilistic approach". A probabilistic approach includes a strong mathematical background; Therefore, it can be applied to the power grid to examine the system status system [13]-[16]. Probabilistic approaches may be applied to the evaluation of the distributed generation. Instead of acquiring some measurement, the result with the deterministic load flow with extreme values of the input file, the probabilistic load flow approach (PLF) can solve the random problem of the DG distribution network. These types of studies give a more general picture and complete stress effects with respect to the deterministic feedstock approach. Studies [17] says that the singular values of the cover image are modified after applying DWT with the singular values of the watermark image. Author analysed proposed ABC optimization algorithm is applied to a standard 14-bus and 57- bus system [18]. Author discussed about implematation of quadratic programming (QP) when applied for 33 bus system [19]. Crowsearch algorithem [20] used to check the robustness of the system statistical analysis has been done which shows the robustness of the algorithm. Y P Obulesu discussed based on observation that voltage profile at the buses is improved after installing two DGs by decreasing the power losses of the system to 2.1681MW [21]. Research based on identification of location of DGs using Index Vector method [22]. The proposed technique demonstrated on 15 bus RDN. Author discussed about weighting method and penalty function approach for location of DG [23]. Placement of DG and sizing along with capacitor is analyzed with DLF method [24].

\section{RESEARCH METHOD}

The key objective of this work is to analyze the probabilistic load flow applied to the distribution system and plan the location and sizing of DGs for extreme loss saving. The distribution system is considered to be a radial system with Wind-based DG connected to it and with uncertain load demand. This includes probabilistic modeling of: Power injected by Wind-based DG. Uncertain load demand on each bus. The various tools used for the evaluation of the problem are: Backward-forward sweeping load-flow (BFSL) algorithm and artificial bee colony (ABC) algorithm.

\subsection{Distribution generation}

Distribution Generations are currently increasing an increasing interest of all actors in the electricity market. In recent years, of course, thanks to economic, ecological and political reasons, the standard installation, characterized by the production of central mass energy and long, long transmission networks is still supported by the energyResources to the distribution network, a suitable common folded as a distributed generation (DG). It should be added that in recent years, DG penetration has become in distribution systems in a manner faster that achieves the maximum limit of the approved connections in many countries, which are managed by the, approach to the standard passive networks. Therefore, over the years, it approaches structure networks, design and function must radically be modified in an extremely manner, while the generation should be considered as an integral part of the distribution network system. Investments and important research are then needed to the electrical Renew distribution sector, achieve a kind of "self-healing" 
distribution system that not only allows management of large groups of energy resources, but also. , Increased level of service quality. Distributed generation (DG) is not a new notion, but it is a growing technique to provide energy to the skill system's heart. It is primarily dependent on the installation and operation of a limited portfolio of electrical electricity units, compact and clean up in order to close an electric charge (client).

Distributed generation (DG) will be increasingly important in an extremely exponent environment for various reasons: (a) the buyer can provide the buyer in the planning and development of independence installation and flexibility. This is particularly important for sensitive and sophisticated loads in environments subject to interruptions and decreases. (b) The energy costs generated by DG decrease competitive with electricity supplied by the grid. Electricity companies can generate critical points in the grid, especially nearby expenses. This leads to significant savings within the electrical distribution infrastructure, especially extensions. (c) allows an independent electricity generation of a consumer, possibly a less expensive rate, which saves the calculation of utility. Excess energy is published in the commodity market and obtained with a profit. (d) With reductive costs, independent energy producers can install the generation and connect them to the system grid to sell energy with profits. (e) The potential file is to provide a number of titles identified as profit results. Independent energy generators can also provide these services as consumers with an independent generation.

Before entering a distributed generation scheme, a transmission definition or a minimum of a functional frame of the distributed generation must be placed. In general, he prescribed that any integrated power generation technology in the distribution systems complies with the distributed generating umbrella. The names "distributed" and "dispersed" are used interchangeably. You can also classify dispersed generation technologies as renewable and not similar distributors.

Renewable technologies include: (i) solar, photovoltaic or thermo- (ii) geothermal and ocean wind. Nominal cabin technologies include: (i) Combustion engine (ICE) (ii) Combined cycle (iii) Combustion turbine (iv) Micro-Turbine (v) Fuel cell. The distributed generation must not be confused with renewable production. Also distributed generation technology is renewable or not; After all, some distributed production technologies could contribute significantly to current pollution problems if it is completely used.

\subsection{Problem formulation}

The DG's position and dimensions can result in a significant loss of power and a lower voltage profile. It is not recommended to enter the network's DG of very high capacity. The DGS placed in the network must have a total capacity that is consumable inside the distribution network. Any attempt to build highcapacity DG resources in order to export power to the opposite station (inverted energy flow from the substation) results in a significant loss. The network configuration and loads of the distribution system play a critical role in determining the ideal size of the DG for the lowest system loss. The application is formulated to meet two goals. First select a suitable location where the loss of the system becomes minimal; seconds calculates the optimum DG size for the minimum system loss.

This segment presents the proposal for a probabilistic definition for a question of the organization of the LDC with the meaning of the studio framework. The justification of the proposed strategy is to force the approval model for the tasting of trials at the deterministic conditions of the ideal force (OPF). Thus, the measurement of the dynamic/receiving force of the force conditions (3.9 - 3.10) is adapted to the entire sum of circumstances. For each Express, the entry of DG Breeze DG has modified the units of age the age of age, while the trouble of the Bureau is detected and therefore weighted with the likelihood of the event of this circumstance during the full year to estimate liveliness disaster. The ideal distributions of DG elements are not installed by stone, for each operating state complete energy disasters are limited without the fact that frame limits are abused.

The following assumptions are made in order to construct an appropriate planning approach that determines the ideal fuel mixture for renewable DG units:

- The same bus can be connected to more than one type of DG.

- The power factor of all DG units is the same.

Why Because distribution systems often involve multiple locations, the location has little manner on the production authority of wind and solar DG.

\subsection{Objective function}

Under all operational conditions, the purpose of the planning formulation is to reduce annual energy losses in the distribution system.

$$
\text { Minimise Losses }=\sum_{g=1}^{N} P_{\text {lossg }} \times P\left(C_{g}\right) \times 8760
$$


Where $\mathrm{P}_{\text {lossg }}$ is total power losses in the organization during state $\mathrm{g}$.

\subsection{Constraints}

\subsubsection{Power flow equations}

$$
\begin{aligned}
& P_{G g, 1}+\sum_{t=1}^{m} C(g, t) \times P_{D G t, i}-C(g, m+1) \times P_{D i}=\sum_{j=1}^{n} V_{g, i} \times V_{g, j} \times Y_{i j} \times \cos \left(\theta_{i j}+\right. \\
& \left.\delta_{g, j}-\delta_{g, i}\right) \forall i, j, g \ldots \\
& Q_{G g, 1}-C(g, m+1) \times Q_{D g, i}=-\sum_{j=1}^{n} V_{g, i} \times V_{g, j} \times Y_{i j} \times \sin \left(\theta_{i j}+\delta_{g, j}-\delta_{g, i}\right) \forall i, j, g \ldots
\end{aligned}
$$

where

$P_{D G t, i}$ - The rated power of the $t^{\text {th }}$ wind-based DG that is connected to bus $i$.

$\mathrm{P}_{\mathrm{Gg}, \mathrm{i}}$ - Bus i received active power from the substation.

$\mathrm{Q}_{\mathrm{Gg}, \mathrm{i}}$ - Bus i received reactive electricity from the substation.

PDi- Bus i has the high active load.

QDi - Bus i has the high reactive load.

$\mathrm{Vg}, \mathrm{i}-\mathrm{Bus}$ I has the voltage during state $\mathrm{g}$.

$\mathrm{n}$ - The overall quantity of buses in the system.

\subsubsection{Power loss equations}

$$
\begin{aligned}
& P_{\text {loss } g}=0.5 \times \sum_{i=1}^{n} \sum_{j=1}^{n} G_{i j} \times\left[\left(V_{g, i}\right)^{2}+\left(V_{g, j}\right)^{2}-2 \times V_{g, i} \times V_{g, j} \times \cos \left(\delta_{g, j}-\right.\right. \\
& \left.\left.\delta_{g, i}\right)\right] \forall g, i, j
\end{aligned}
$$

\subsubsection{Branch current equations}

$$
I_{g, i j}=\left|Y_{i j}\right| \times\left[\left(V_{g, i}\right)^{2}+\left(V_{g, j}\right)^{2}-2 \times V_{g, i} \times V_{g, j} \times \cos \left(\delta_{g, j}-\delta_{g, i}\right)\right]^{1 / 2} \quad \forall g, i, j
$$

where $I_{g, j i j}$ is the current in the feeder connecting busses $i$ and $j$ during state $g$.

\subsubsection{Slack bus voltage and angle (assumed to be bus 1)}

$$
\begin{aligned}
& V_{g, 1}=1.0 \\
& \delta_{g, 1}=0.0^{\circ}
\end{aligned}
$$

\subsubsection{Voltage limits at load buses}

$$
0.90 \leq V_{g, i} \leq 1.10 \quad \forall i \notin \text { substationbusesandg }
$$

\subsubsection{Feeder capacity limits}

$$
0 \leq I_{g, i j} \leq I_{i j \max }
$$

\subsubsection{Discrete size of wind-based DG}

$$
P_{D G t, i}=a_{t, i} P_{r} \quad \forall i \in B \text { and } t \in m
$$

where

$\mathrm{a}_{\mathrm{i}}$ - The integer variable.

$\mathrm{P}_{\mathrm{r}}$ - The one-column matrix of length $\mathrm{m}$ includes all ratings of the available wind turbines.

B - The set of candidate buses to connect DGs. 


\subsubsection{Maximum penetration on each bus}

$$
\sum_{t=1}^{m} P_{D G t, i} \leq P_{\text {bus }} \quad \forall i
$$

Where $\mathrm{P}_{\mathrm{bus}}$ is the maximum allowable penetration on each bus.

\subsubsection{Maximum penetration of DG units in the system} based DG.

The maximum penetration limit will be calculated based on the average penetration of the wind-

$$
\sum_{i=1}^{n} \sum_{t=1}^{m} C F \times P_{D G t, i} \leq x \times \sum_{i=1}^{n} P_{D i}
$$

where $\mathrm{x}$ denotes the maximum penetration, limit expressed as a percentage of peak load.

\subsection{Artificial bee colony algorithm}

The research behavior of the bee inspired the artificial algorithm of the bee colony. This approach is a stochastic constructive optimization method for extremely obvious and resilient consumption consumption. The bee artificial colony is divided by the ABC algorithm into three categories: used APIs, passers-by and scouts. Weights the APIs represent half of the colony while passers-by are formed from the other half. The amount of quantity of each food source symbolizes the quality (fitness) of the associated solution, since the position of a power source refers to a solution that is possible on the optimization problem.

The number of employees corresponds to the number of food sources. When the APIs leave a power source, the previously used bee becomes a scout. First, the ABC procedure would create an initial population that is discriminated against by case $\mathrm{P}(\mathrm{C}=0) \mathrm{SNO}$ food situations, where $\mathrm{Sn}$ is the size of the food sources. Each $\mathrm{Xi}$ solution $(\mathrm{i}=1,2,3,3, \ldots, \mathrm{Sn})$ could be a dimension carrier called the number of optimization parameters. After initialization, the solution population is acc $=1.2 .3$ MCN of the state research of API work of API work and scout. A used bee can change the answer to your store based on local facts and test the value of the new source fitness. If the new has a greater physical shape than the previous one, the dedicated bee learns the new and forgets the old man. Otherwise, you remember the location of the previous one. If all the APIs used have completed the search, inform the pendrants on the nectar levels and restaurants. An observer evaluates the nectar data released by all the APIs used and selects a power source with a probability that corresponds to the quantity of quantity. The observer can modify the point of memory and examine the nectar of the quantity of the source of the candidates and the rented bee.

If the amount of nectar is greater than the previous one, the bee will learn the new position and forget the old woman. An observer selects a nutritional source fully based on the probability value associated with the supply source associated with the following formulawhefites whose physical value value of the IITH solution based on the amount of nectar of the I-TH position. The ABC could use the following modification form to create a new food position from the previous one. When $\mathrm{K}$ and $\mathrm{J}$ generate random clues, but $\mathrm{K}$ should differ from I. can handle the availability of a replacement power of Xij and use a bee to visually view the comparison of two food products. As can be seen from the equations mentioned above, thus reducing the difference between the parameters of the parameters $\mathrm{Xij}$ and $\mathrm{XKJ}$, thus disturbed on the $\mathrm{Xij}$ position. As a result, the size of the tempests is reduced when searching for the optimal solution in the search room.

The abandoned food source of bees would have been replaced by a new food search source by Scouts. The behavior of the feed search is emulated in the ABC algorithm by randomly generating a power outlet and generating an exchange of a disconnected plug with a replacement base. If a competitive advantage can no longer be increased after a number of cycles, the nutritional source must be abandoned. The default number of cycles called "limit" for the task of the ABC algorithm is a critical control parameter. If the source specified and $\mathrm{J}$ is, the scout finds a new food source to replace it. This method is often defined because it generates every new VIJ source position, which evaluates the artificial bee and compares its physical shape with the previous one. If the new source of food is so good or better than the old man, the old man can be replaced in its memory. Otherwise, he holds the old man in his memory. As you can see from the previous explanation, the $\mathrm{ABC}$ algorithm has three critical control parameters: the number of food sources $(\mathrm{SN})$, the value of the limit "" and the maximum number of cycle $(\mathrm{MCN})$.

a. Begin by populating the population of solutions xij, $I=1,2, \ldots ., S N, j=1,2, \ldots ., D$.

b. Assess the population's fitness.

c. Set the Cycle to 1

d. Develop new xij solutions for the employed bees and assess their fitness

e. For the employed bees, use the greedy selection process.

Optimal placement and sizing of distributed generation for loss minimization using ... (Madhu Valavala) 
f. For each solution Vij, calculate the probability values Pij.

g. From the solutions xi picked betting on Pi, create fresh solutions xij for observers and assess their fitness.

h. For the spectator bees, use the greedy selection technique.

i. Identify an abandoned response for the scout and replace it with a randomly generated solution xij.

j. Write down the most efficient solution.

k. Cycle is equal to Cycle+1.

1. Repeat steps (4) through (11) until Cycle $=\mathrm{MCN}$ is reached.

m. Show the best solution.

\section{RESULTS AND DISCUSSION}

The artificial bee colony algorithm was tested on the bus systems IEEE33 and IEEE118 to see how effective it is. The ABC optimization method was used in the MATLAB 7.1 software for this problem, and all programs were performed on a $3.00 \mathrm{GHz}$ GB, Intel Core 2 Duo PC RAM. The following were measured as test cases:

Case 1: In this case, the GD unit to provide only an active supply, ie the power factor of the unit.

Case 2: In this case, the DG unit to generate active energy that reactivates the maintenance of a power factor of 0.8. These two test cases are carefully examined, and the results are compared and contrasted with $\mathrm{ABC}$ optimization.

\subsection{Constant model}

In this model, both the load demand on the network and the power output of the DGs to be installed are considered to be constant.

\subsubsection{Results for 33 bus radial distribution system}

The proposed technique is being tested using the standard IEEE 33-bus system as a first example. The system's total actual and reactive power loads are 3.72MW and 2.3MVAr, respectively. An attempt was made to deploy DG at different locations in order to examine the effect of DG placement at multiple locations.

\section{Single DG placement and Two DG Placement}

The maximum size of single DG unit is fixed as 3.5 MVA. The test results for both cases are as Figure 1 and Figure 2.

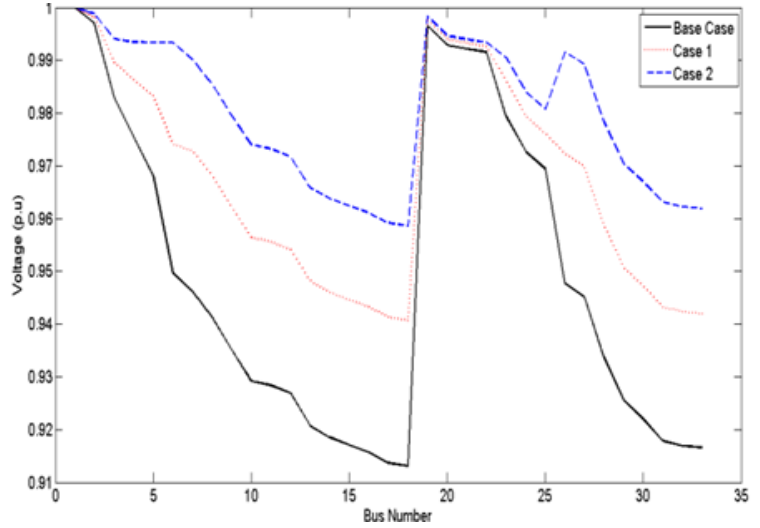

Figure 1. Voltage Profile of 33 bus system for single DG placement

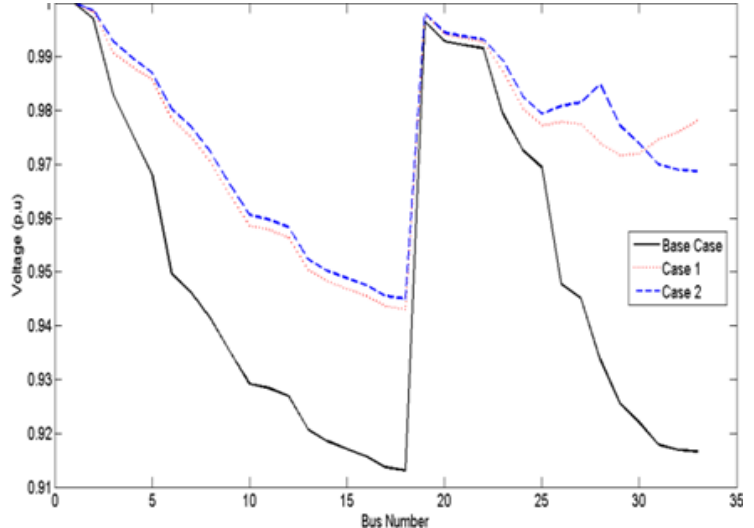

Figure 2. Voltage Profile of 33 bus system for two DG placement

The load-flow technique calculated system real power losses to be $202.66 \mathrm{~kW}$, with a minimum voltage of 0.9131 p.u. The true power losses in Case 1 and Case 2 after the installation of a single DG were found to be $103.97 \mathrm{~kW}$ and $61.58 \mathrm{~kW}$, respectively. Even the voltage magnitudes improved, with minimum values of 0.9407 p.u and 0.9586 p.u in Case 1 and Case 2, respectively. 
The load-flow technique calculated system real power losses to be $202.66 \mathrm{~kW}$, with a minimum voltage of 0.9131 p.u. The real power losses in Case 1 and Case 2 after the installation of two DGs were found to be $85.99 \mathrm{~kW}$ and $30.19 \mathrm{~kW}$, respectively. Even the voltage magnitudes improved, with minimum values of 0.9403 p.u and 0.9450 p.u in Case 1 and Case 2, respectively. Figures 1 and 2 demonstrate the voltage profile of a 33 bus system with a single DG placement and a double DG placement, respectively.

\subsubsection{Results for a radial distribution system with 118 buses}

An IEEE 118-bus system is used as another example for evaluating the suggested method's efficacy. The system's total actual and reactive power loads are 22.71GW and 17.04GVAr, respectively.

The load-flow technique calculated system real power losses to be $1298.38 \mathrm{~kW}$, with a minimum voltage of 0.8688 p.u. The real power losses in Case 1 and Case 2 after the installation of a single DG were found to be $1017.01 \mathrm{~kW}$ and $921.72 \mathrm{~kW}$, respectively. Even the voltage magnitudes improved, with minimum values of 0.9053 p.u and 0.9053 p.u in Case 1 and Case 2, respectively.

The load-flow technique calculated system real power losses to be $1298.38 \mathrm{~kW}$, with a minimum voltage of 0.8688 p.u. After the commissioning of two DGs, the real power losses in Cases 1 and 2 were determined to be $805.55 \mathrm{~kW}$ and $587.51 \mathrm{~kW}$, respectively. Even the voltage magnitudes improved, with minimum values of 0.9095 p.u and 0.9053 p.u in Case 1 and Case 2, respectively. Figure 3 and Figure 4 demonstrate the voltage profiles of the 118 bus system for single DG placement and double DG placement, respectively.

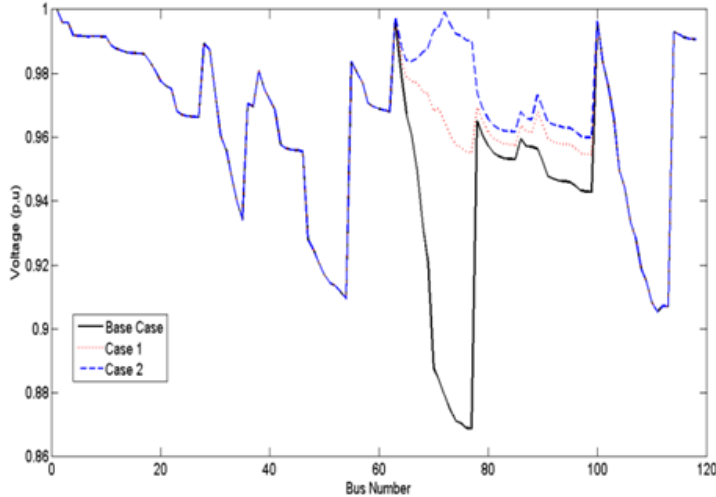

Figure 3. Voltage Profile of 118 bus system for single DG

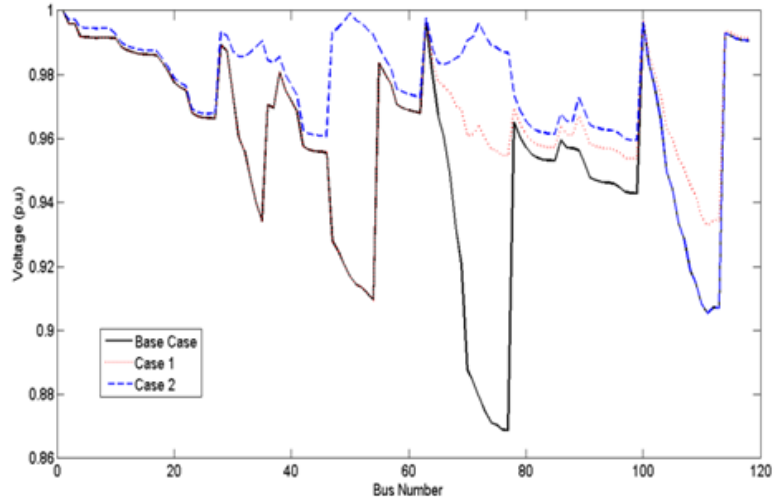

Figure 4. Voltage Profile of 118 bus system for two DG

\section{CONCLUSION}

The artificial bee colony approach is used in this study to suggest a probabilistic solution for the optimal placement and sizing of several distributed generations. The results were confirmed using IEEE-33 and IEEE-118 bus radial distribution test equipment. In this study, two scenarios were examined: one with only real power injection and the other with both real and reactive power injection. With the installation of DGs, we see a reduction in the overall real power loss of the system in both scenarios. When the DGs are controlled to inject both actual and reactive power, the loss reduction is observed to be greater. The probabilistic approach shows that the sizes of the DGs to be installed are reduced for almost the same percentage loss reduction when compared with the situation where the load and DG output are considered to be constant. In this work, IEEE test systems have been considered to validate and prove the substance of the proposed probabilistic approach. This can be extended to live rural distribution feeders and can be utilized successfully to reduce the burden on the substation as well as on the transmission line.

\section{REFERENCES}

[1] Y. M. Atwa, and E. F. El-Saadany, "Probabilistic approach for optimal allocation of wind-based distributed generation in distribution systems," IET Renew, Power Gener., vol. 5, no. 1, pp. 79-88, 2011, doi: 10.1049/ietrpg.2009.0011

[2] F. S. Abu-Mouti and M. E. El-Hawary, "Optimal distributed generation allocation and sizing in distribution systems via artificial bee colony algorithm," in IEEE Transactions on Power Delivery, vol. 26, no. 4, pp. 20902101, Oct. 2011, doi: 10.1109/TPWRD.2011.2158246.

[3] J. O. Kim, S. W. Nam, S. K. Park, and C. Singh "Dispersed generation planning using improved Hereford Ranch algorithm," Electric Power Systems Research, vol. 47, no. 1, pp. 678-683, 1998. 
[4] Gandomkar, M. Vakilian and M. Ehsan, "Optimal distributed generation allocation in distribution network using Hereford Ranch algorithm," 2005 International Conference on Electrical Machines and Systems, 2005, pp. 916-918 vol. 2, doi: 10.1109/ICEMS.2005.202678.

[5] T. Griffin, K. Tomsovic, D. Secrest and A. Law, "Placement of dispersed generation systems for reduced losses," Proceedings of the 33rd Annual Hawaii International Conference on System Sciences, 2000, pp. 9 pp.-, doi: 10.1109/HICSS.2000.926773.

[6] K. Nara, Y. Hayashi, K. Ikeda and T. Ashizawa, "Application of tabu search to optimal placement of distributed generators," 2001 IEEE Power Engineering Society Winter Meeting. Conference Proceedings (Cat. No.01CH37194), 2001, pp. 918-923 vol.2, doi: 10.1109/PESW.2001.916995.

[7] J. A. Greatbanks, D. H. Popovic, M. Begovic, A. Pregelj and T. C. Green, "On optimization for security and reliability of power systems with distributed generation," 2003 IEEE Bologna Power Tech Conference Proceedings, 2003, pp. 8 pp. vol. 1-, doi: 10.1109/PTC.2003.1304111.

[8] T. K. A. Rahman, S. R. A. Rahim and I. Musirin, "Optimal allocation and sizing of embedded generators," Proceedings. National Power and Energy Conference, 2004, pp. 288-294, doi: 10.1109/PECON.2004.1461660.

[9] G. Celli and F. Pilo, "Optimal distributed generation allocation in MV distribution networks," PICA 2001. Innovative Computing for Power - Electric Energy Meets the Market. 22nd IEEE Power Engineering Society. International Conference on Power Industry Computer Applications (Cat. No.01CH37195), 2001, pp. 81-86, doi: 10.1109/PICA.2001.932323.

[10] G. Celli and F. Pilo, "MV network planning under uncertainties on distributed generation penetration," 2001 Power Engineering Society Summer Meeting. Conference Proceedings (Cat. No.01CH37262), 2001, pp. 485-490 vol.1, doi: 10.1109/PESS.2001.970074.

[11] G. Carpinelli, G. Celli, F. Pilo and A. Russo, "Distributed generation siting and sizing under uncertainty," 2001 IEEE Porto Power Tech Proceedings (Cat. No.01EX502), 2001, pp. 7 pp. vol. 4-, doi: 10.1109/PTC.2001.964856.

[12] R. Karki and R. Billinton, "Cost-effective wind energy utilization for reliable power supply," in IEEE Transactions on Energy Conversion, vol. 19, no. 2, pp. 435-440, June 2004, doi: 10.1109/TEC.2003.822293.

[13] N. Acharya, P. Mahat, and N. Mithulananthan, "An analytical approach for DG allocation in primary distribution network," International Journal of Electrical Power and Energy Systems, vol. 28, no. 10, pp. 669-678, Dec. 2006, doi. 10.1016/j.ijepes.2006.02.013.

[14] M. P. Selvan and K. S. Swarup, "Distribution system load flow using object-oriented methodology," 2004 International Conference on Power System Technology, 2004, pp. 1168-1173 Vol.2, doi: 10.1109/ICPST.2004.1460178.

[15] A. Silvestri, A. Berizzi and S. Buonanno, "Distributed generation planning using genetic algorithms," PowerTech Budapest 99. Abstract Records. (Cat. No.99EX376), 1999, pp. 257-, doi: 10.1109/PTC.1999.826689.

[16] C. Grigg et al., "The IEEE Reliability Test System-1996. A report prepared by the reliability test system task force of the application of probability methods subcommittee," in IEEE Transactions on Power Systems, vol. 14, no. 3, pp. 1010-1020, Aug. 1999, doi: 10.1109/59.780914.

[17] B. P. Kulkarni, S. Sai Krishna, K. Meenakshi, P. Kora and K. Swaraja, "Performance analysis of optimization algorithms GA, PSO, and ABC based on DWT-SVD watermarking in OpenCV Python environment," 2020 International Conference for Emerging Technology (INCET), 2020, pp. 1-5, doi: 10.1109/INCET49848.2020.9154134.

[18] R. Deshmukh and A. Kalage, "Optimal placement and sizing of distributed generator in distribution system using artificial bee colony algorithm," 2018 IEEE Global Conference on Wireless Computing and Networking (GCWCN), 2018, pp. 178-181, doi: 10.1109/GCWCN.2018.8668633.

[19] L. Fajariyanti, R. S. Wibowo, O. Penangsang, D. F. U. Putra and N. K. Aryani, "optimal placement and sizing distributed generation (DG) considering energy storage using ABC-QP algorithm," 2019 International Seminar on Intelligent Technology and Its Applications (ISITIA), 2019, pp. 126-131, doi: 10.1109/ISITIA.2019.8937204.

[20] A. K. Pandey and S. Kirmani, "Placement of distributed generator in distribution systems using Crow search algorithm," 2019 International Conference on Electrical, Electronics and Computer Engineering (UPCON), 2019, pp. 1-6, doi: 10.1109/UPCON47278.2019.8980157.

[21] G. S. Rao and Y. P. Obulesh, "ABC and ANN based minimization of power loss for distribution system stability," 2015 IEEE 10th Conference on Industrial Electronics and Applications (ICIEA), 2015, pp. 772-777, doi: 10.1109/ICIEA.2015.7334213.

[22] M. Dixit, P. Kundu and H. R. Jariwala, "Optimal placement and sizing of DG in Distribution system using Artificial bee colony algorithm," 2016 IEEE 6th International Conference on Power Systems (ICPS), 2016, pp. 1-6, doi: 10.1109/ICPES.2016.7584010.

[23] S. Biswas, A. Chatterjee and S. K. Goswami, "An artificial bee colony based optimal placement and sizing of distributed generation," Proceedings of The 2014 International Conference on Control, Instrumentation, Energy and Communication (CIEC), 2014, pp. 356-360, doi: 10.1109/CIEC.2014.6959109.

[24] A. K. Tudu and A. Bhaumik, "A comparative approach of PSO and ABC algorithm for optimal positioning and capacity sizing of DG and Shunt Capacitor in a Distribution network using DLF algorithm," 2018 Second International Conference on Computing Methodologies and Communication (ICCMC), 2018, pp. 276-280, doi: 10.1109/ICCMC.2018.8487996. 